\title{
Characterizing and Correcting Phase Biases in Short-Term, Multilooked Interferograms
}

\author{
Yasser Maghsoudi ${ }^{1}$, Andrew J. Hooper ${ }^{1}$ and Tim J. Wright ${ }^{1}$, Milan Lazecky ${ }^{1}$ and Homa \\ Ansari $^{2}$ \\ ${ }^{1}$ COMET, School of Earth and Environment, University of Leeds, LS2 9JT, UK. \\ ${ }^{2}$ Remote Sensing Technology Institute (IMF), German Aerospace Center (DLR). \\ Corresponding author: Yasser Maghsoudi (y.maghsoudi@leeds.ac.uk)

\section{Key Points: InSAR, Phase Bias, Fading Signal, Correction}

\section{Abstract}

Interferometric Synthetic Aperture Radar (InSAR) is widely used to measure deformation of the

Earth's surface over large areas and long time periods. A common strategy to overcome coherence loss in long-term interferograms is to use multiple multilooked shorter interferograms, which can cover the same time period but maintain coherence. However, it has recently been shown that using this strategy can introduce a bias (also referred to as a "fading signal") in the interferometric phase, particularly over vegetated areas. We isolate the signature of the phase bias by constructing daisy chain sums of short-term interferograms covering identical 1-year time periods, but using interferograms of different time spans. This shows that the shorter interferograms are more affected by this phenomenon and the degree of the effect also depends on ground cover types. We, propose a method for correcting the phase bias, based on the assumption that the bias in an interferogram is linearly related to the sum of the bias in shorter interferograms spanning the same time. We tested the algorithm over a study area in western Turkey by comparing average velocities against results from a phase linking approach that has been shown to be almost insensitive to the phase bias. Our corrected velocities agree well with those from phase linking approach. Our approach can be applied to global compilations of short-term interferograms and offers the 
possibility of accurate long-term velocities without a requirement for coherence in long-term

27 interferograms.

\section{Introduction}

Interferometric Synthetic Aperture Radar (InSAR) is a powerful tool for monitoring ground deformation associated with earthquakes, volcanoes, landslides, and anthropogenic activities (e.g. Biggs et al. 2009; Foroughnia et al. 2019; Juncu et al. 2017; Massonnet et al. 1995; Temtime et al. 2018; Walters et al. 2011; Weiss et al. 2020). The accuracy of the estimated deformation is traditionally thought to depend on uncorrected tropospheric and ionospheric delays, errors in phase unwrapping, uncertainties in knowledge of satellite position, phase decorrelation due to changes in scattering behavior between successive images, and system noise. Most of these error terms are associated with individual epochs and cancel out when calculating the wrapped loop closure phase at full spatial resolution, $\Delta \varphi$, defined for three epochs $(\mathrm{i}, \mathrm{j}, \mathrm{k})$ as:

$$
\Delta \varphi_{i, k}=\left|\varphi_{i, k}-\left(\varphi_{i, j}+\varphi_{j, k}\right)\right|_{2 \pi}
$$

where $\varphi_{i, j}$, for example, is the phase difference for a pixel in the interferogram between epochs $\mathrm{i}$ and $\mathrm{j}$, and ||$_{2 \pi}$ indicates that the result is given modulo $2 \pi$ (i.e. wrapped) (Michaelides et al. 2019; Zwieback et al. 2016).

For full-resolution processing, the wrapped loop closure phase must be zero. If multilooking, or other forms of spatial filtering, is carried out as part of the processing, then $\Delta \varphi$ will not be precisely equal to zero. This is because the filtering adds a term to each interferogram, the aim of which is reduce the noise term, which does not cancel in the closure phase calculation. This is not an issue for applications provided that the expected value of this term is zero. However, De Zan et al. 


\section{The paper is a non-peer reviewed EarthArXiv preprint}

(2015) showed that the expected value of the filtering term is non-zero for some ground cover types.

Nonzero closure phases are a product of the spatial filtering and are mainly associated with the scattering and electrical properties of the ground surface (De Zan et al. 2015; Michaelides et al. 2019). Previous studies have suggested that changes in soil moisture and in the water content of vegetation might lead to these phase inconsistencies (De Zan and Gomba 2018; De Zan et al. 2014). Although the amount of the bias caused by such inconsistencies is small in each individual interferogram, its accumulation in time can significantly impact the final estimated velocities, particularly for applications where millimetric accuracy is required. Ansari et al. (2021) showed short-interval multilooked interferograms are more impacted by this phenomenon and referred to this effect as the fading signal due to its short-lived nature. This is particularly problematic for time-series analysis approaches that exploit pixels where coherence can only be maintained for short time intervals - these pixels are likely to be strongly impacted by phase bias.

Mitigation strategies that have been proposed include correcting interferograms using physical models such as a moisture-induced phase model (De Zan and Gomba 2018) or using "phase linking" (PL) approaches, described below. Due to the varied sources of the phase bias, employing a single physical moisture-induced phase model cannot account for all possible sources of phase inconsistencies and no generic model exists to incorporate all possible sources of the phase bias (Ansari et al. 2021). PL approaches, on the other hand, can effectively mitigate this phenomenon by incorporating all possible $N(N-1) / 2$ interferometric phases obtained from $N$ SAR acquisitions (Guarnieri and Tebaldini 2008). The key step in the PL approaches is to optimally estimate single-master phases for each pixel from all possible interferometric combinations. These methods retrieve maximum available information in InSAR data stacks 


\section{The paper is a non-peer reviewed EarthArXiv preprint}

(Samiei-Esfahany et al. 2016). Though efficient and robust, PL approaches require a large number of interferograms and are computationally expensive, particularly for systems like Sentinel-1, where there might be several hundred acquisitions. Moreover, the quality of the PL estimated phases highly depends on the availability of the long-term interferograms. In case of the decorrelated regions, the applicability and practicality of PL methods is limited.

In this contribution, we first explore the characteristics of the phase bias by investigating its temporal and spatial behavior. We then develop and test an empirical mitigation strategy to correct short-term interferograms for the phase bias. Correcting for the phase bias in the short-term interferograms is of great importance, in particular when the Small BAseline Subset (SBAS) algorithms e.g. (Berardino et al. 2002; Morishita et al. 2020) are being used.

Our approach assumes that there is a linear relationship between the bias in a single interferogram and the sum of the biases in the shorter interferograms spanning the same time. Employing this assumption, we can estimate bias corrections for each interferogram through a linear least squares inversion. We demonstrate the effectiveness of the proposed mitigation strategy by comparing the resultant velocities with the phase linking approach.

\section{Study site}

We chose a study area in the west of Turkey that has a variety of ground cover types, including forested and agricultural areas where long-term coherence is difficult to maintain (Figure 1). Spatial heterogeneity in the land cover allows us to investigate the bias effect in these different land covers ranging from more coherent urban areas to the agricultural and forest areas. The area is imaged by Sentinel-1 A and B data on every overpass. We processed all interferometric pairs 


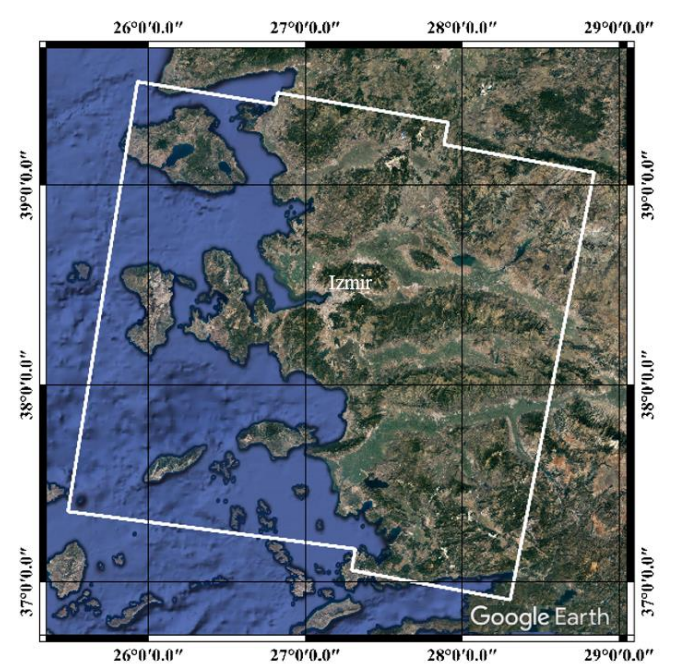

(a)

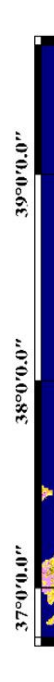

Figure 1) Study site: (a) Overview of the study area located in the western Turkey. Izmir is the major city, situated along the Aegean coast. The white polygon shows the footprint of the Sentinel-1 data from descending path 36. (b) Land cover map obtained through the Copernicus Land Monitoring Service (https://lcviewer.vito.be).

from one-year of Sentinel-1 acquisitions on track 36, where 60 images were acquired in the period spanning 1 February 2017 to 31 January 2018. All interferograms were generated using the automated workflows from the COMET-LiCSAR system (Lazecký et al. 2020), and were multilooked by factors of 5 in the range and 20 in the azimuth directions and geocoded onto a 100 m grid using elevation data from the Shuttle Radar Topography Mission (Farr et al. 2007).

\section{Phase bias characterization}

Although the bias in individual interferograms cannot be isolated, we can measure phase bias in sets of interferograms by examining loop closure phases using different combinations of data.

Figure 2 shows how we calculate the closure phase using a set of multilooked interferograms in a loop. In this example, we subtracted the sum of three 6-day interferograms (b), (c) and (d) from an 18-day interferogram (a) to isolate the loop closure phase (e). We use the notation $\Delta \varphi^{18-6}=18$ day $-\sum_{1}^{3}(6$ day $)$ to denote this loop closure phase. For the rest of this paper 
107

108

109

110

111

112

113

114 115 m days.

116

117

118

119

120

\section{1}

122

123

124

125

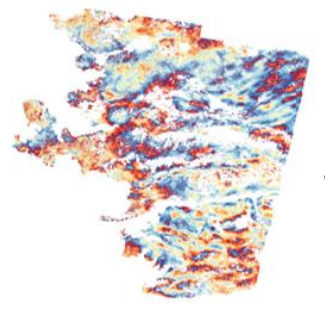

(a)

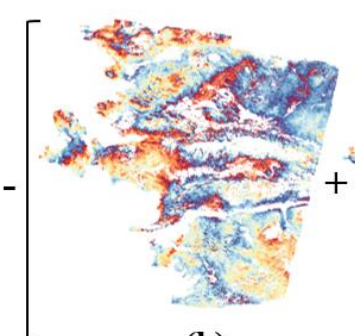

(b)

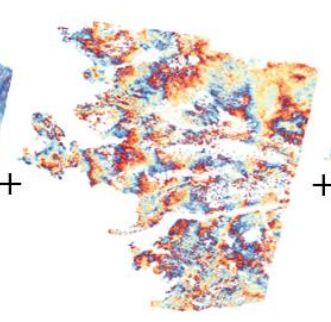

(c)

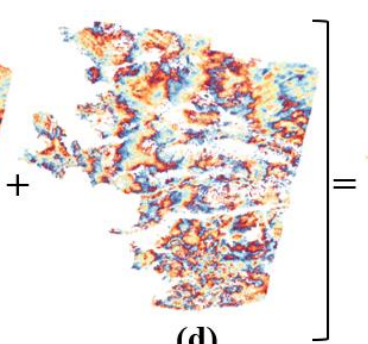

(d)

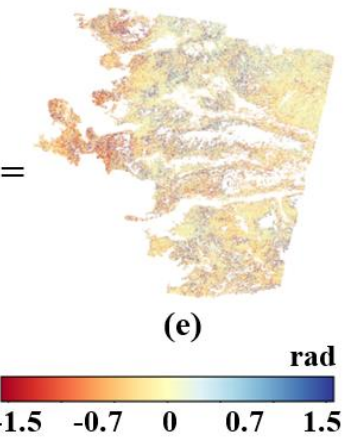

Figure 2) Example of closure phase calculated from an 18-day interferogram and three 6-day interferograms. 18-day interferogram (a) spans 2017-02-18 to 2017-03-08. Three 6-day interferograms (b,c and d) span 2017-02-18 to 2017-02-24 (b), 2017-02-24 to 2017-03-02 (c) and 2017-03-02 2017-03-08 (d). The resulting closure phase is shown in (e).

$\Delta \varphi^{n-m}$ indicates the loop closure phase from subtracting the summation of all $m$ day interferograms from a $n$ day interferogram spanning the same time. We also used the notation $\sum_{360 \text { days }} \Delta \varphi^{n-m}$, for example, to show the 360-day cumulative loop closure phase calculated as the difference between 360-day "daisy chain" sums of interferograms with length n days and

We use wrapped phases throughout this study to calculate the closure phases, with the result of any phase differences rewrapped to the interval $\pm \pi$. Taking a closer look at Figure 2(e), we can see a spatially correlated signal that varies across the image. Comparing this Figure with Figure 1(b), this phase bias signal appears strongest in the vegetated areas.

To understand how the phase bias varies in interferograms of different lengths, we calculated the 360-day cumulative loop closure phase using $n=60$ and $m=6,12,18,24,30$ and 36 . The results are shown in Figure 3(top).

The results show that shorter interferograms are more affected by this phenomenon, with cumulative loop closure phases reducing in size dramatically as the length of the shorter interferograms in the loop increases. This observation agrees with the effect of the fading signal 
126 (Ansari et al. 2021). The magnitude of the bias averaged over multiple pixels strongly depends

127 on the ground cover type, with cropland and forested pixels having significantly larger bias than

128 urban pixels (Figure 3 (bottom)).

129

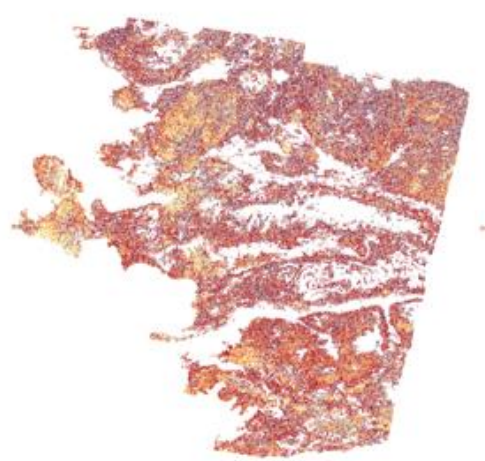

$\mathrm{m}=6$

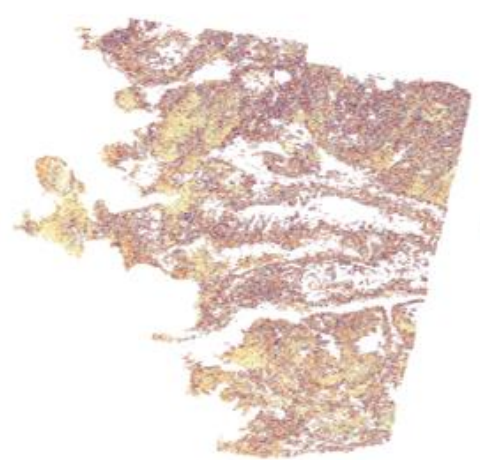

$\mathrm{m}=24$

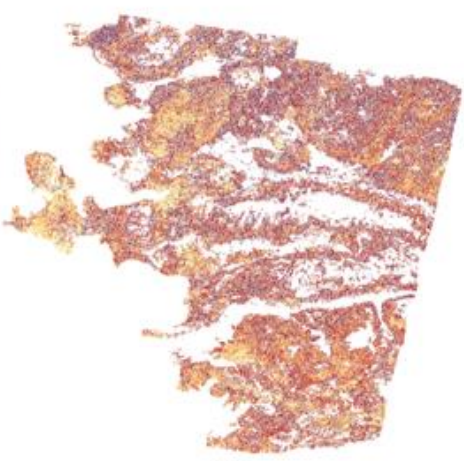

$\mathrm{m}=12$

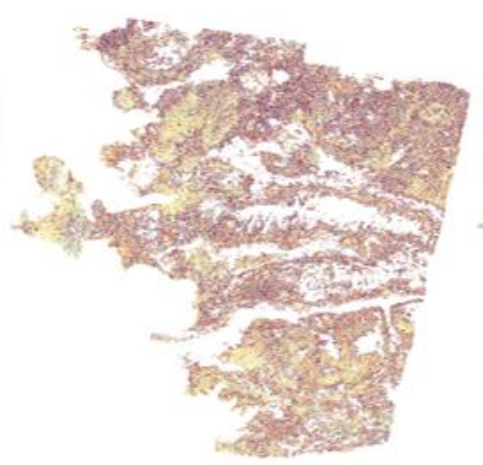

$\mathrm{m}=30$

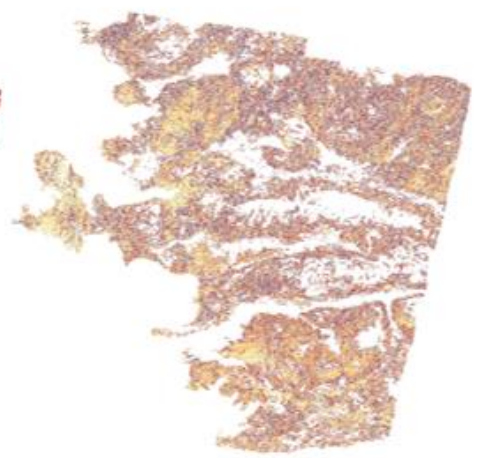

$\mathrm{m}=18$

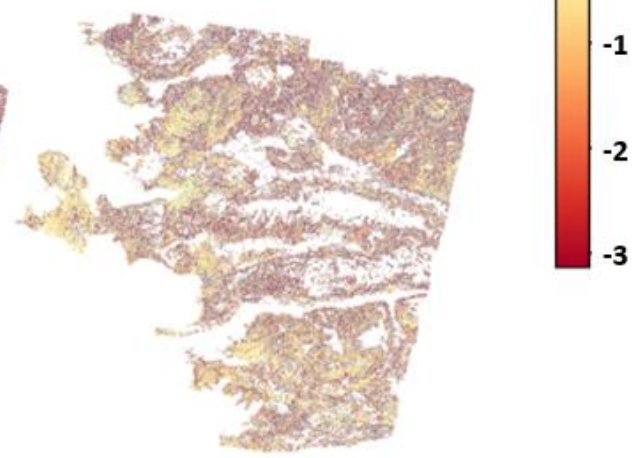

$\mathrm{m}=36$

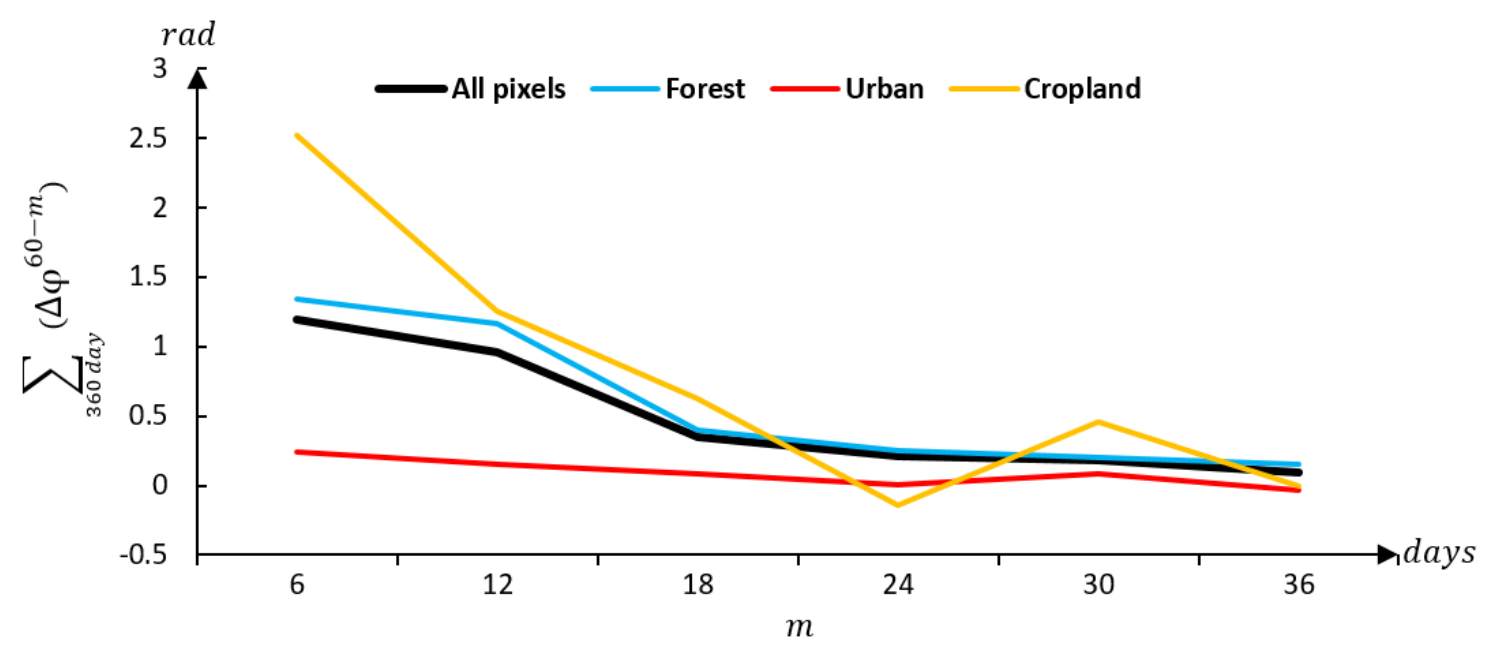

130

131 Figure 3) 360-day cumulative loop closure phases, $\sum_{360 \text { day }}\left(\Delta \varphi^{60-m}\right)$, for varying timespans, $m$ (top), and mean value of

132 cumulative loop closure phases for different land cover classes as a function of $\mathrm{m}$ (bottom) 
133 To test how the phase bias accumulates in time, we calculated $\sum_{i=1}^{t}\left(\Delta \varphi^{18-6}\right)_{i}$ for $\mathrm{t}=1, \ldots, 20,20$

134 being the total number of consecutive 18 day interferograms in the 360-day observation period

135 (Figure 4). The results show that although the amount of the closure phase is small in each

136 individual loop, it increases with time. The rate of bias accumulation is not steady throughout the

137 year, being highest for cropland and lowest for urban pixels.

138

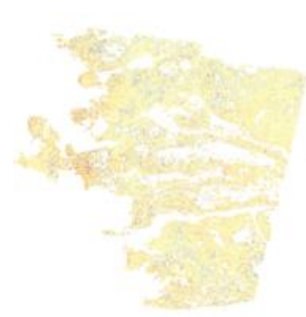

$\mathrm{t}=2$

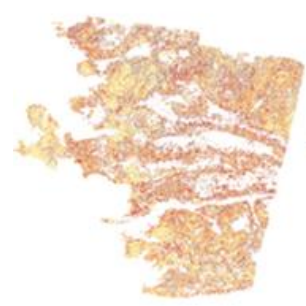

$\mathrm{t}=12$

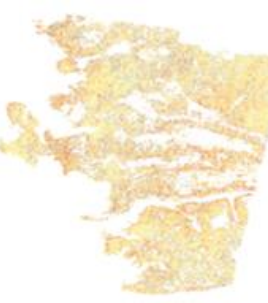

$\mathrm{t}=4$

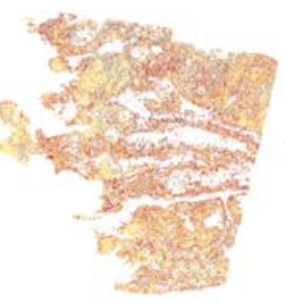

$\mathrm{t}=14$

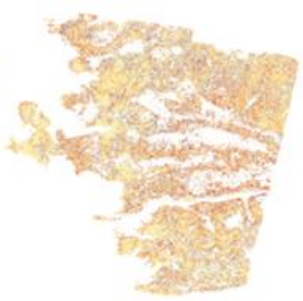

$\mathrm{t}=6$

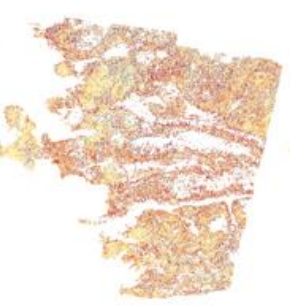

$\mathrm{t}=16$

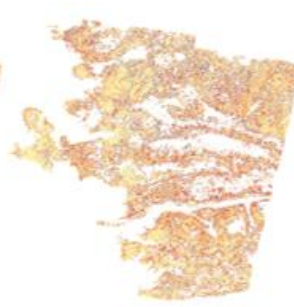

$t=8$

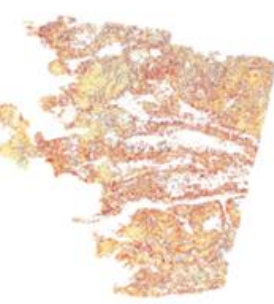

$t=18$

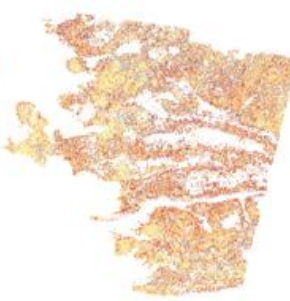

$\mathrm{t}=10$

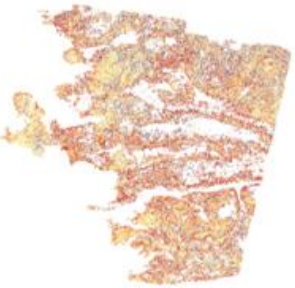

$t=20$
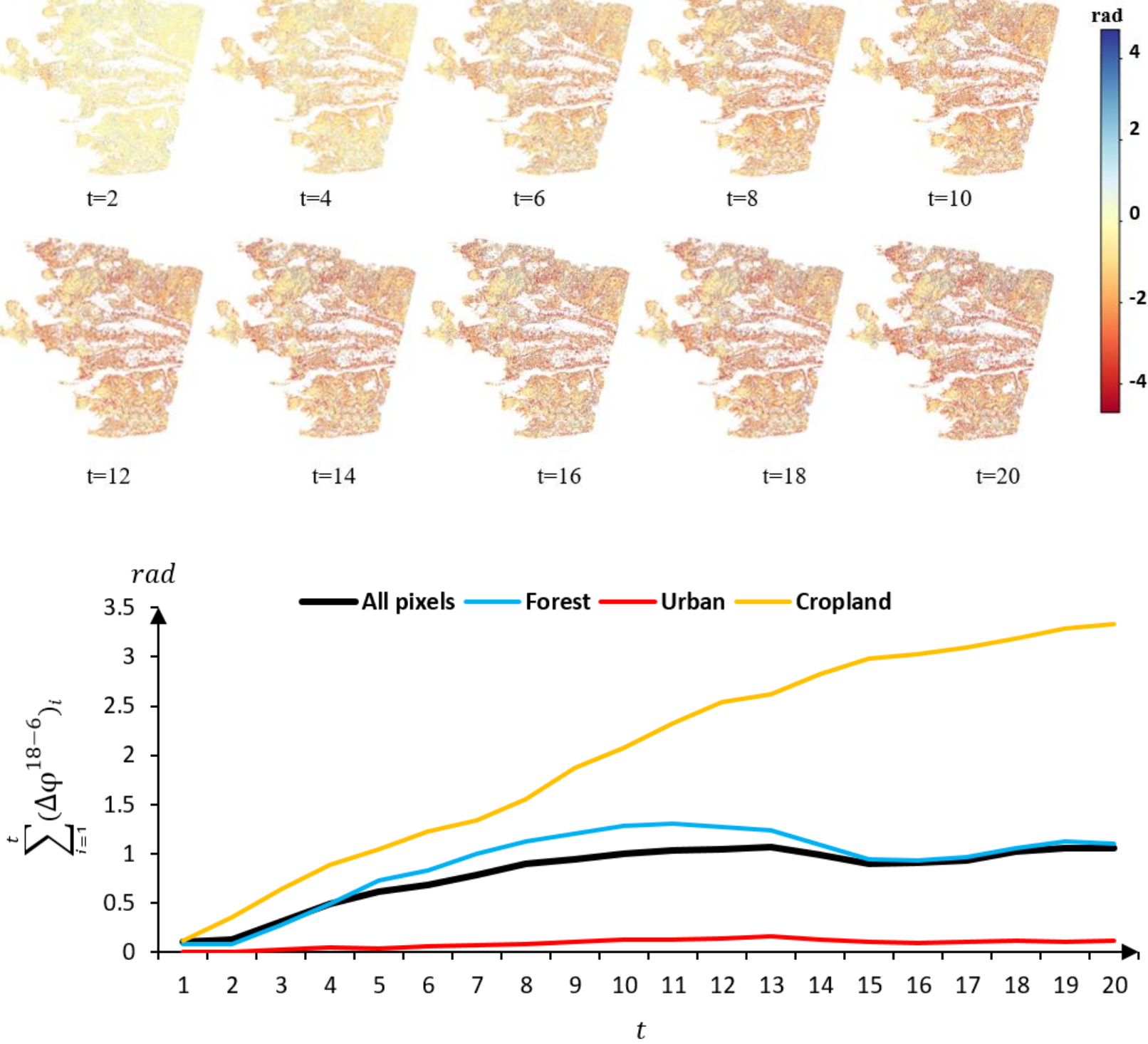

139

140 Figure 4) The temporal accumulation of the loop closures obtained by $\sum_{i=1}^{t}\left(\Delta \varphi^{18-6}\right)_{i}$ (top), and temporal accumulation of phase

141 bias averaged for different land covers within the scene (bottom). 
142 We also investigated the temporal variation in bias accumulation by calculating $\Delta \varphi^{60-6}$ loop

143 closure phases covering different two-month periods (Figure 5). Each plot in Figure 5 (top)

144 belongs to a two-month period. Figure 5 (bottom) illustrates the mean value of plots in different

145 periods and in different landcovers. The plots indicate that the strength of the bias varies

146 throughout the year. The largest mean value of phase bias is observed in the first plot, which

147 corresponds to the period February and March. The smallest mean value, on the other hand,

148 occur in late summer (August to September).

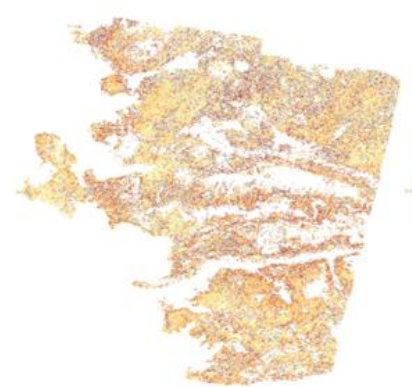

Feb-Mar

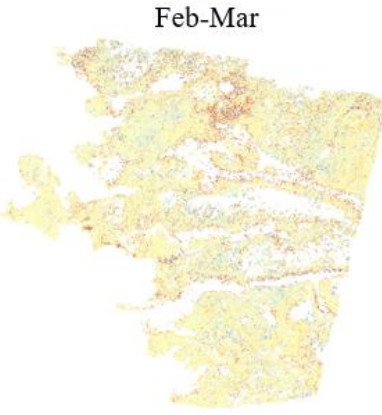

Aug-Sep

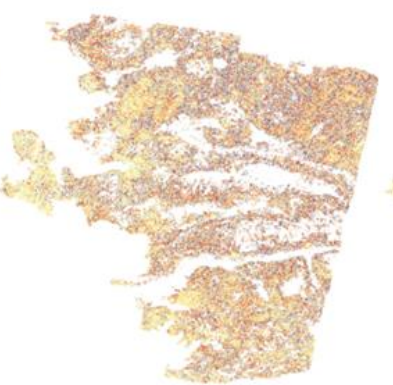

Apr-May

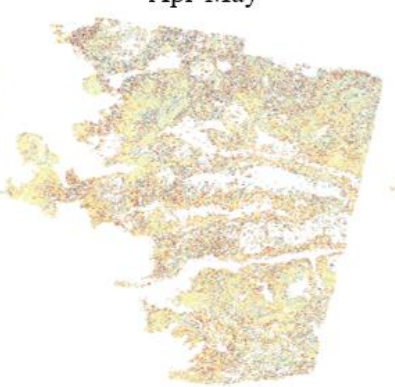

Oct-Nov

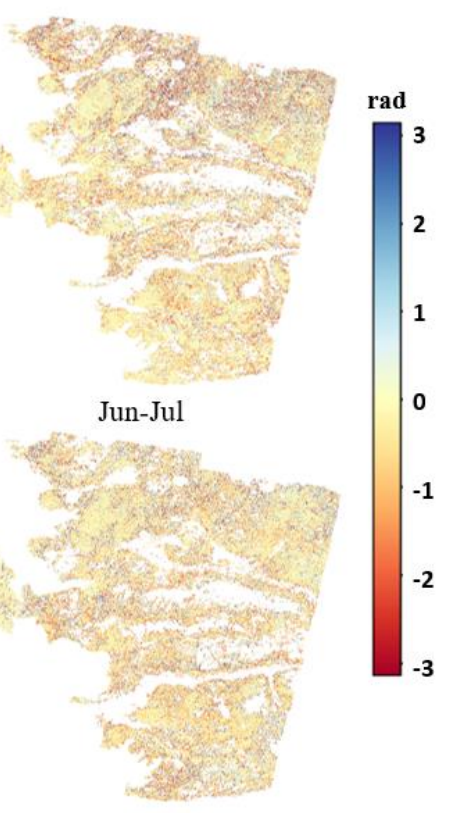

Dec-Jan

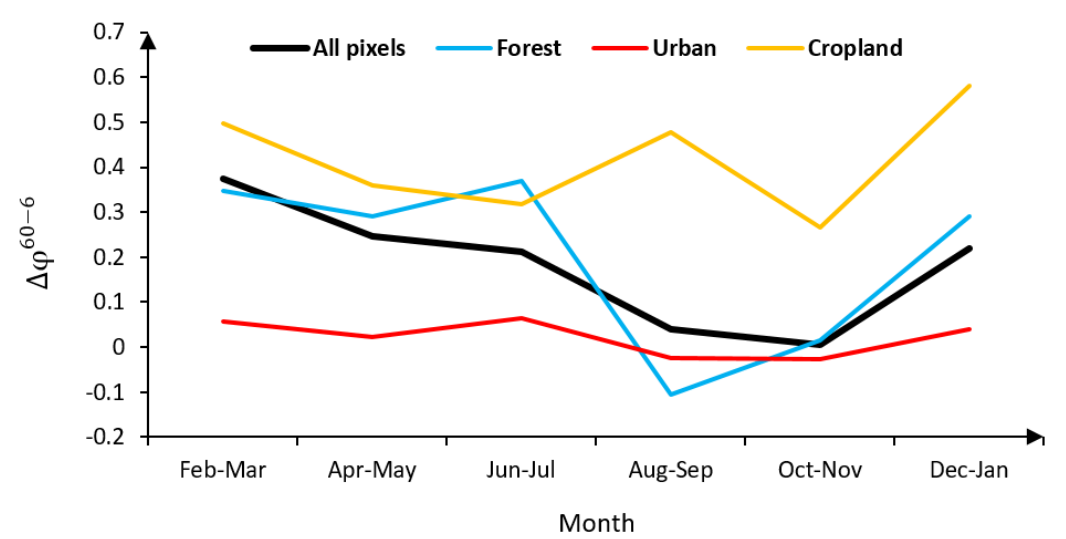




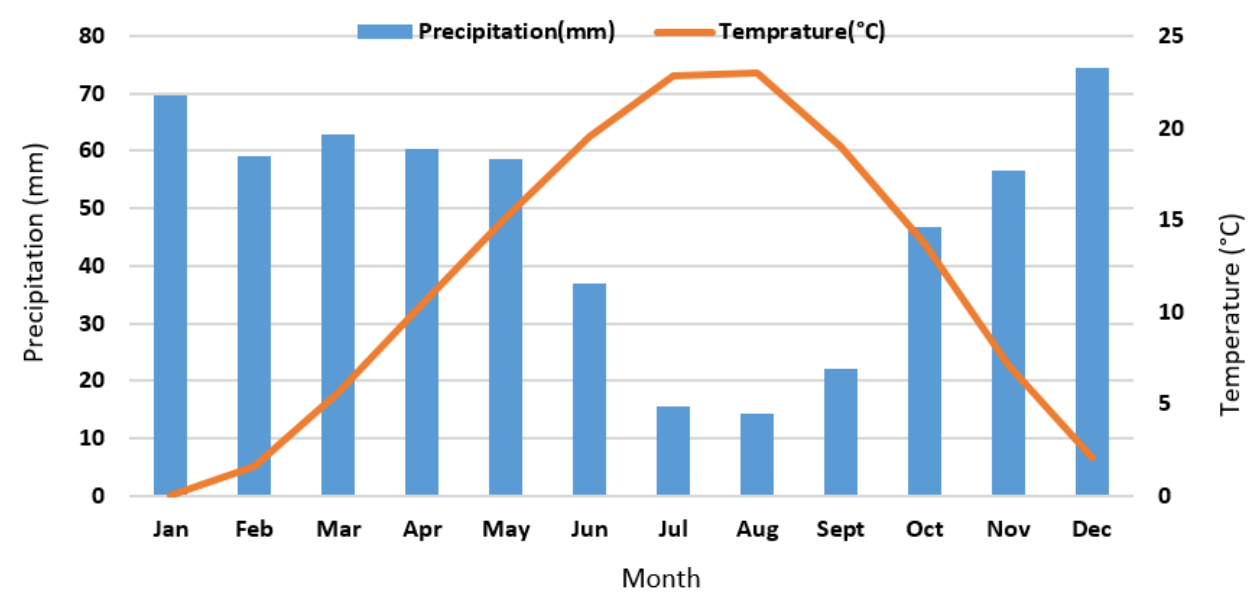

152

153

154

Figure 6) Average monthly temperature and rainfall of Turkey for 1991-2020 obtained from https://climateknowledgeportal.worldbank.org/country/turkey/climate-data-historical

This matches well with the precipitation season in the west coast of Turkey (highest in January to March and lowest in July to September) as shown in Figure 6. The closure phase in cropland pixels is more complex and may depend on several factors, including the vegetation growth as well as moisture variation.

Finally, in the last experiment of this section, we investigated the effect of the adaptive phase filtering (Goldstein and Werner 1998) on phase bias. Phase filtering is commonly applied to interferograms to reduce phase noise which greatly improves phase unwrapping performance. We applied a spatial filter to the multilooked interferograms using an adaptive power spectrum filter with FFT window size $=32$ and alpha $=1$. Figure 7 compares the cumulative loop closure phase $\sum_{360 \text { day }}\left(\Delta \varphi^{18-6}\right)$ using unfiltered and filtered interferograms. Filtering increases the mean value of the loop closure phase (the bias), by effectively increasing the multilooking factor. Therefore, we recommend caution in using filtered interferograms for time-series analysis. 
169

170

171

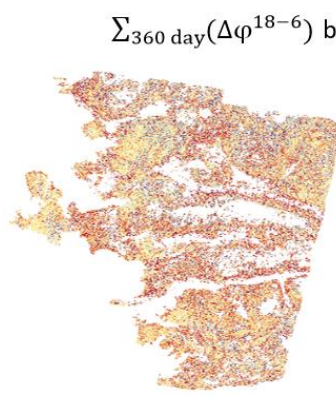

(a) (b). Here we used the adaptive Goldstein filtering.

\section{Phase bias correction} interferograms. including:

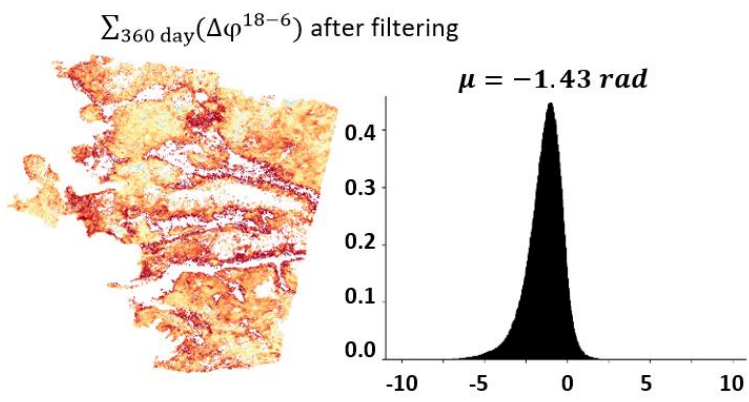

(b)

Figure 7) $\sum_{360 \text { day }}\left(\Delta \varphi^{18-6}\right)$ and its histogram applied to the multilooked interferograms that are not filtered (a) and those that are

In the COMET-LiCSAR automatic processing system (Lazecký et al. 2020), interferograms have been processed that connect each epoch, i, to the three or four nearest acquisitions in time, backward and forward. We therefore aim to develop a bias correction approach that uses just the interferograms formed from the closest three connections so that accurate velocities can be obtained without requiring mass processing of large numbers of additional longer-term

Several loop closure phases can be calculated for an individual pixel, from these interferograms,

$$
\begin{gathered}
\Delta \varphi_{i, i+2}=\varphi_{i, i+2}-\left(\varphi_{i, i+1}+\varphi_{i+1, i+2}\right) \text { and } \\
\Delta \varphi_{i, i+3}=\varphi_{i, i+3}-\left(\varphi_{i, i+1}+\varphi_{i+1, i+2}+\varphi_{i+2, i+3}\right)
\end{gathered}
$$

182 where $\Delta \varphi_{i, i+2}$ and $\Delta \varphi_{i, i+3}$ are the $\Delta \varphi^{12-6}$ and $\Delta \varphi^{18-6}$ loop closure phases respectively. Assuming 183 the closure phase is due to biases and noise in each interferogram, Equation (2) and (3) can be 184 written as:

$$
\Delta \varphi_{i, i+2}=\delta_{i, i+2}-\left(\delta_{i, i+1}+\delta_{i+1, i+2}\right)+\varepsilon \text { and }
$$




$$
\Delta \varphi_{i, i+3}=\delta_{i, i+3}-\left(\delta_{i, i+1}+\delta_{i+1, i+2}+\delta_{i+2, i+3}\right)+\varepsilon,
$$

185

186

187

188

189

190

191

192

193

194

$$
\begin{gathered}
\delta_{i, i+2}=a_{1}\left(\delta_{i, i+1}+\delta_{i+1, i+2}\right) \text { and } \\
\delta_{i, i+3}=a_{2}\left(\delta_{i, i+1}+\delta_{i+1, i+2}+\delta_{i+2, i+3}\right),
\end{gathered}
$$

195 where $a_{1}$ and $a_{2}$ are unknown constants that linearly relate the bias in the longer interferograms

196 to the sum of the corresponding biases in the short interferograms covering the same time period.

197 If we assume that 360-day interferograms have negligible bias, $a_{1}$ and $a_{2}$ can be estimated for

198 each pixel by calculating the ratio of the cumulative loop closure phases for 12- and 6-day

199 interferograms and 18- and 6-day interferograms respectively:

$$
\begin{aligned}
& a_{1}=\frac{\Delta \varphi^{360-12}}{\Delta \varphi^{360-6} \text { and }} \\
& a_{2}=\frac{\Delta \varphi^{360-18}}{\Delta \varphi^{360-6}}
\end{aligned}
$$




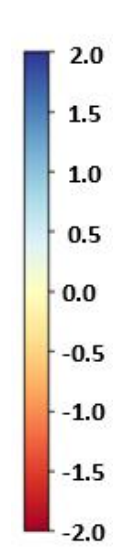

$a_{1}$

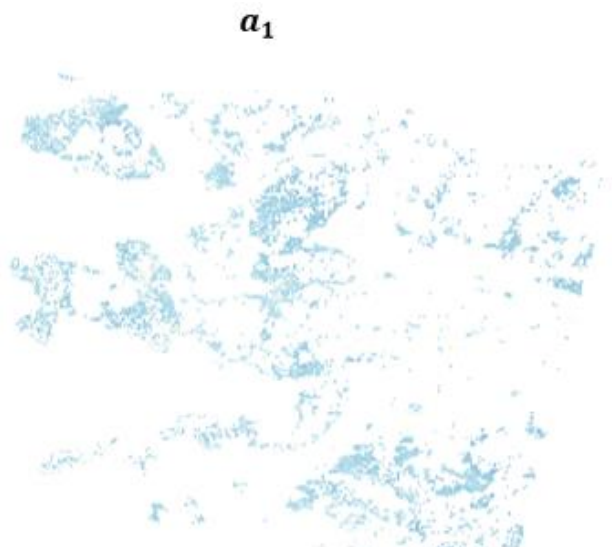

$$
\boldsymbol{\mu}=\mathbf{0 . 4 7}
$$

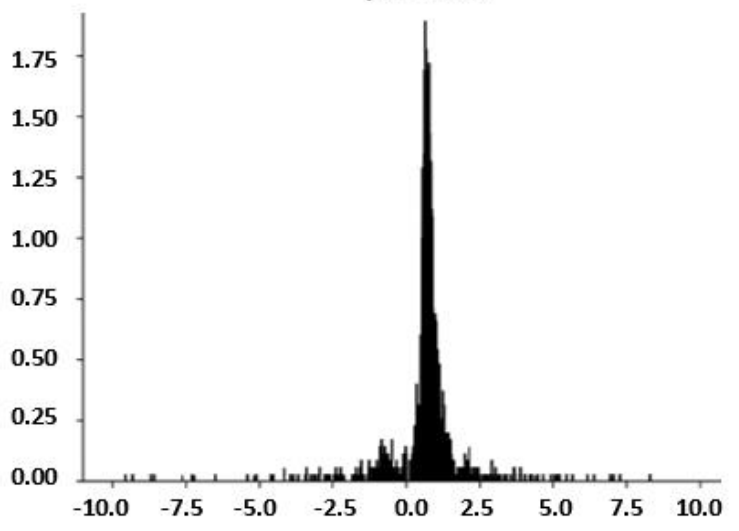

$a_{2}$

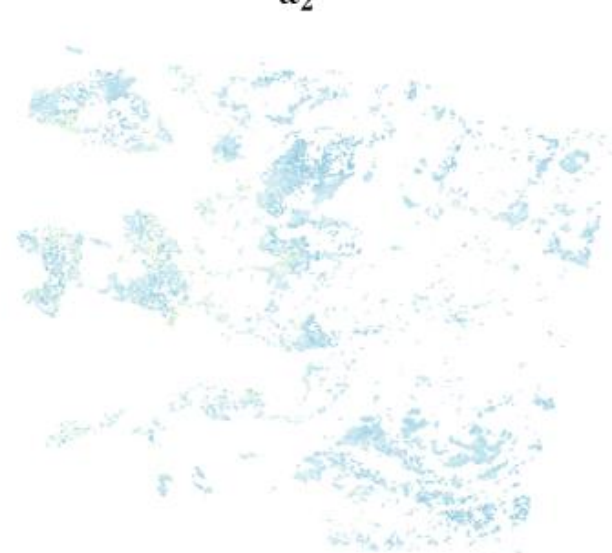

$\boldsymbol{\mu}=\mathbf{0 . 3 1}$

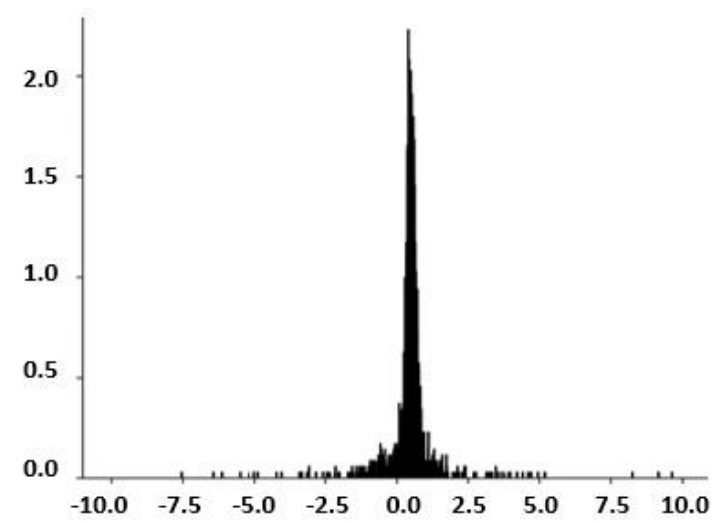

Figure 8 . The maps of $a_{1}$ and $a_{2}$ (top), and their corresponding histograms (bottom)

202 When estimating the regularization parameters $a_{1}$ and $a_{2}$ only pixels that remain coherent for a 203 period of 1 year can be used. Figure 8 shows the maps of the $a_{1}$ and $a_{2}$ and their histograms. 204 Although estimates of $a_{1}$ and $a_{2}$ for each pixel are noisy, there is no systematic pattern in space 205 (Figure 8 (top)), suggesting that a single value is appropriate. In this study, we used the mean 206 values of 0.47 and 0.31 for $a_{1}$ and $a_{2}$ respectively for all pixels.

207 If $a_{1}$ and $a_{2}$ are constants, using equations (4) to (7) and including all observations in epochs $i$ to $208 i+3$ lead to a series of observation equations relating the closure phases to unknowns $\delta_{i, i+1}$.

$$
\left(\begin{array}{c}
\Delta \varphi_{i, i+2} \\
\Delta \varphi_{i+1, i+3} \\
\Delta \varphi_{i, i+3}
\end{array}\right) \cong\left(\begin{array}{ccc}
a_{1}-1 & a_{1}-1 & 0 \\
0 & a_{1}-1 & a_{1}-1 \\
a_{2}-1 & a_{2}-1 & a_{2}-1
\end{array}\right)\left(\begin{array}{c}
\delta_{i, i+1} \\
\delta_{i+1, i+2} \\
\delta_{i+2, i+3}
\end{array}\right)
$$


This reduces the number of unknowns to N-1, the biases for the 6-day interferograms. The system

210 of equations is then overdetermined when $\mathrm{N} \geq 5$ (with $2 \mathrm{~N}-5$ loop closure observations) and the

211 unknown bias terms can be estimated using a linear least squares inversion.

212 Upon the estimation of the bias terms, every single 6-, 12- and 18-day interferogram can then be 213 corrected using

$$
\begin{gathered}
\varphi_{i, i+1}^{c}=\varphi_{i, i+1}-\hat{\delta}_{i, i+1} \text { and } \\
\varphi_{i, i+2}^{c}=\varphi_{i, i+2}-\hat{\delta}_{i, i+2}=\varphi_{i, i+2}-a_{1}\left(\hat{\delta}_{i, i+1}+\hat{\delta}_{i+1, i+2)}\right) \text { and } \\
\varphi_{i, i+3}^{c}=\varphi_{i, i+3}-\hat{\delta}_{i, i+3}=\varphi_{i, i+3}-a_{2}\left(\hat{\delta}_{i, i+1}+\hat{\delta}_{i+1, i+2}+\hat{\delta}_{i+2, i+3}\right),
\end{gathered}
$$

214 where $\varphi_{i, i+1}, \varphi_{i, i+2}$ and $\varphi_{i, i+3}$ are the original 6-day, 12-day and 18-day interferograms and $\varphi_{i, i+1}^{c}$,

$215 \varphi_{i, i+2}^{c}$ and $\varphi_{i, i+3}^{c}$ are the corrected interferograms. The $\hat{\delta}_{i, j}$ are the estimated bias terms.

\section{Correction results}

217 All the experiments in this section were carried out on a set of coherent pixels, which were selected 218 by applying a threshold of 0.3 on the 18-day average coherence. We estimated the corrections 219 using Equations (10) and corrected all the 6-day 12-day and 18-day interferograms covering our 360-day study period using Equations (11), (12) and (13) respectively.

221 Figure 9 shows a comparison between the closure phase $\sum_{360 \text { day }}\left(\Delta \varphi^{18-6}\right)$ using the original 222 interferograms and that found using the corrected interferograms. It is clear that correcting the 223 interferograms has significantly decreased the closure phase, with its mean and the standard 224 deviation decreasing from $-1.05 \pm 2.7 \mathrm{rad}$ prior to correction to $0.03 \pm 1.7 \mathrm{rad}$.

225 We also show (Figure 10) corrected and uncorrected time series of line of sight (LOS) displacement calculated from just the 6-day interferograms for some example points in different 
Figure 9) The cumulative loop closure phase $\sum_{1 y r}(18$ day -6 day) calculated using (a) the original interferograms (b)
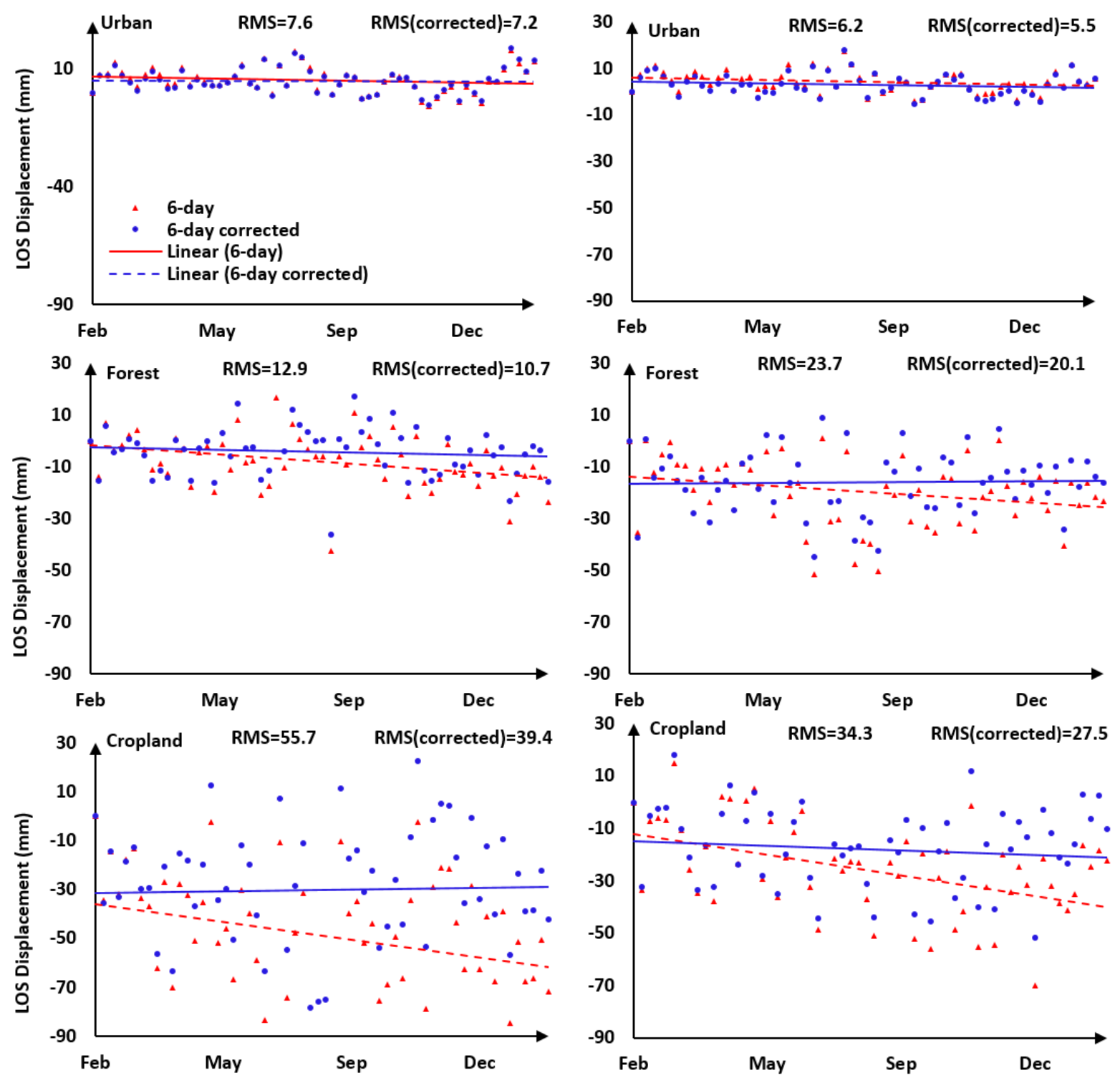

Figure 10) The LOS time-series displacement for two example points in each land cover. RMS and RMS(corrected) show the root-mean-square of residuals calculated before and after correction respectively 


\section{The paper is a non-peer reviewed EarthArXiv preprint}

235 land covers. Pixels in urban areas change the least with the correction, whereas the agricultural

236 pixels have larger values of corrections. We also calculate the root-mean-square (RMS) of the

237 residuals before and after the correction (Figure 10); the correction reduces the scatter of the data

238 for all land cover types. Considering all the pixels, the mean RMS residual of fit to the linear time

239 series model has reduced from 27.2 rad before the bias correction to 20.7 rad after correcting for

240 the phase bias.

\section{Validation}

242 As well as demonstrating the reduction in cumulative loop closure phases, we can also compare

243 line-of-sight velocities estimated from our corrected and uncorrected data with velocities from an

244 approach that is less sensitive to phase bias. We use a Phase Linking (PL) approach for this

245 validation test, which uses all possible interferograms and has been shown to be rather unaffected

246 by phase bias of short-term interferograms (Ansari et al. 2021). There are a number of PL methods

247 in the literature. These methods try to obtain the best estimates of $\mathrm{N}-1$ phase differences for a

248 pixel relative to the primary date using the $\mathrm{N}(\mathrm{N}-1) / 2$ available interferometric phases. PL

249 methods are categorized into maximum-likelihood estimators (Ferretti et al. 2011), least squares

250 estimators (Samiei-Esfahany et al. 2016), Eigen decomposition-based phase estimators (Cao et al.

251 2016; Fornaro et al. 2015) and Eigen decomposition-based Maximum-likelihood estimator (Ansari

252 et al. 2018).

253 Eigen decomposition-based methods are relatively computationally efficient and straightforward

254 to implement; we use the approach from (De Zan et al. 2007; Fornaro et al. 2015), hereafter 
255

Considering $\mathrm{T}$ as the $N$ by $N$ coherence matrix, the Eigen decomposition of $\mathrm{T}$ can be obtained as

$$
\boldsymbol{T}=\sum_{i=1}^{N} \lambda_{i} v_{i} v_{i}^{H}
$$

where the eigenvalues $\lambda_{i}$ are arranged in descending order as $\lambda_{1} \geq \lambda_{2} \geq \ldots, \geq \lambda_{N}$, $v_{i}$ is the corresponding eigenvector associated with eigenvalue $\lambda_{i}$ and $\mathrm{H}$ stands for the conjugate transpose.

Phases $\hat{\varphi}$ are estimated by extracting the phases of the eigenvector associated with the largest eigenvalue. The EPL velocity i.e. $V_{E P L}$ can then be estimated using these linked phases. Full details of the algorithm are described in (De Zan et al. 2007; Fornaro et al. 2015). We used the a posteriori coherence of (Ferretti et al. 2011) as a quality measure for phase estimation. In this study, we chose a value of 0.4 as a threshold for the a posteriori coherence to mask out the unreliable phases.

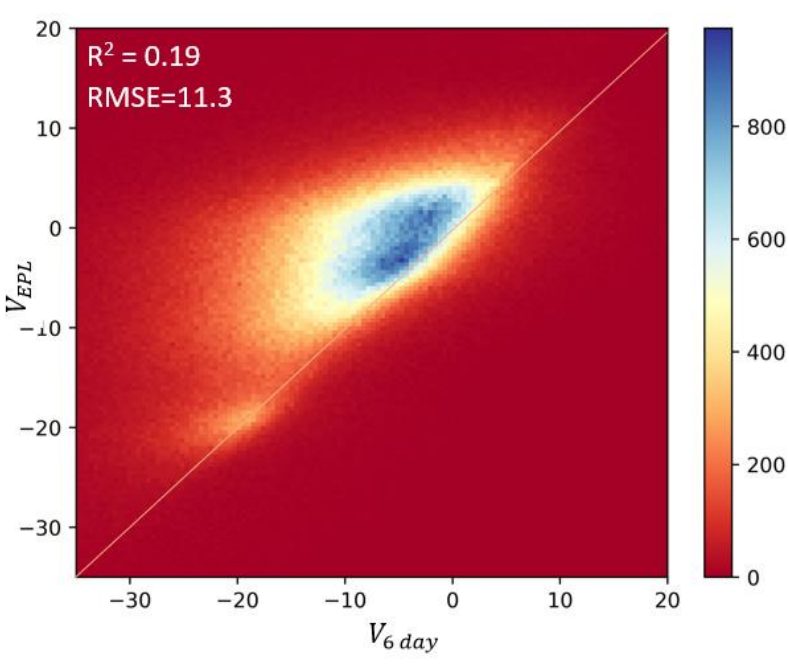

(a)

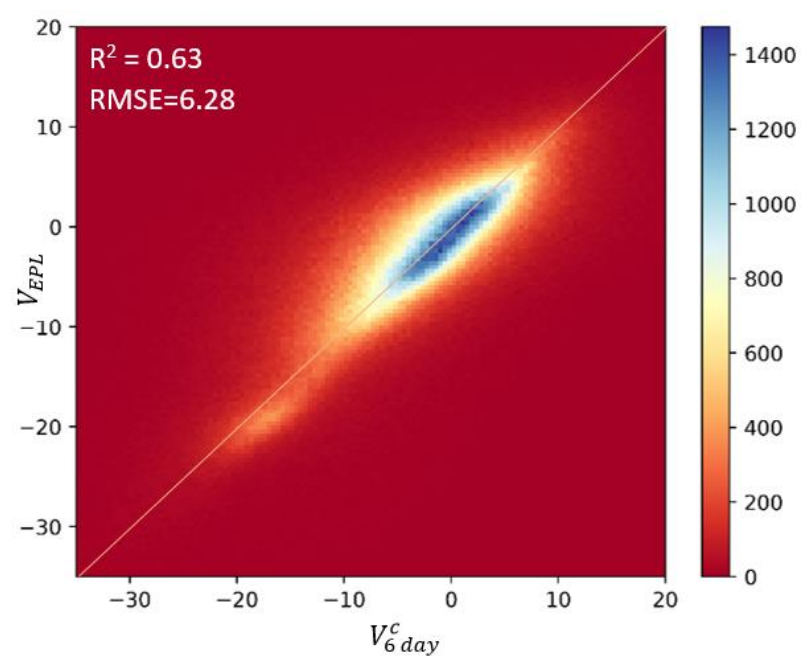

(b)

Figure 11) Scatterplot of 360-day velocities obtained from Eigen decomposition Phase Linking $\left(V_{E P L}\right)$ against velocities obtained 


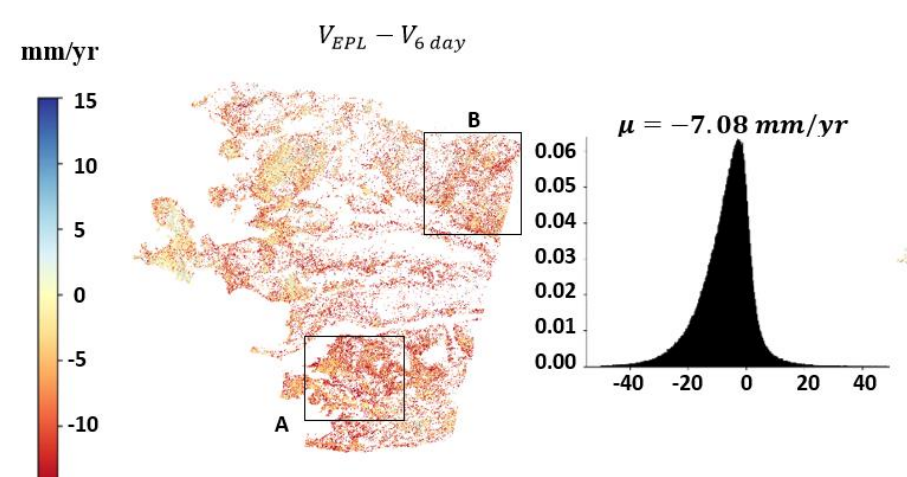

(a)

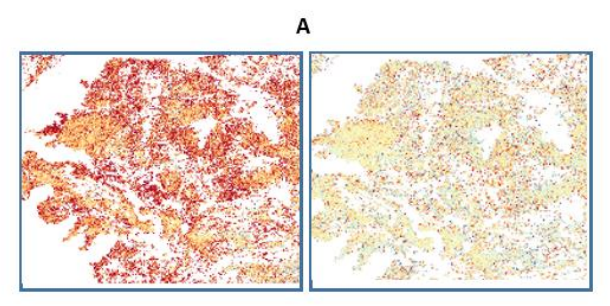

(c)

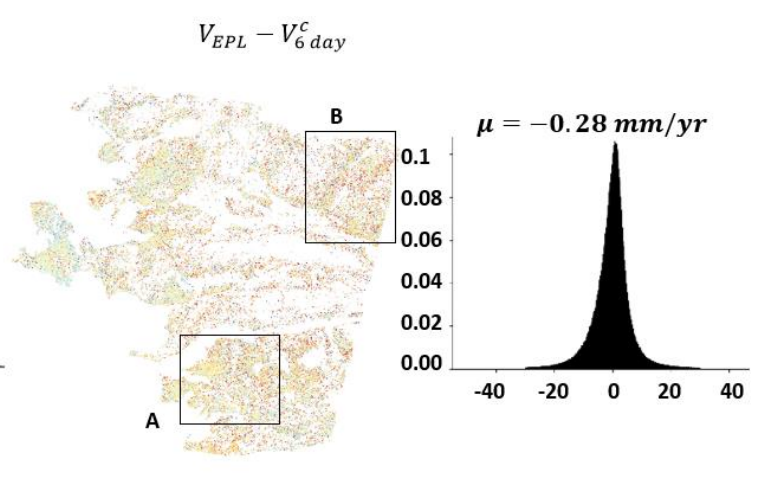

(b)

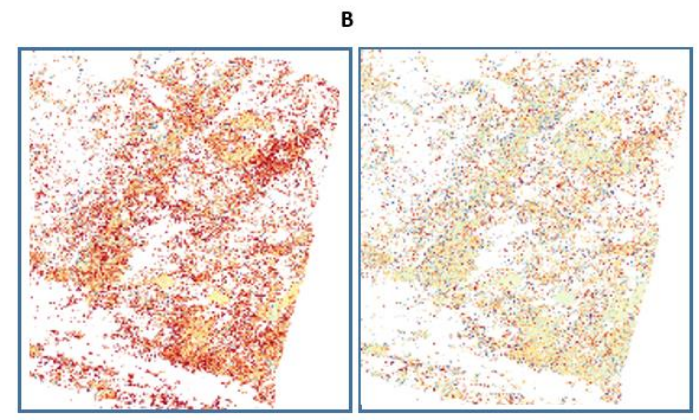

(d)

Figure 12) Effect of the phase bias correction on the velocity estimation. Difference between the EPL velocity and (a) the uncorrected 6-day velocity i.e. $V_{E P L}-V_{6 \text { day }}$, (b) corrected 6-day velocity i.e. $V_{E P L}-V_{6 \text { day }}^{c}$ are shown as maps and histograms. (c,d) zoomed-in views of the two subsets for areas A and B.

We calculated velocities from our corrected and uncorrected 6-day interferograms over the 360day time period and checked the effectiveness of our bias correction strategy by comparing our estimated velocities before and after correction with the EPL velocities (Figures 11, 12).

A scatterplot of the original 6-day estimated velocities for all pixels in our Turkey frame, $V_{6}$ day, versus the velocities from EPL, $V_{E P L}$, is skewed to left (Figure 11 (a)), indicating that the velocities for many of the pixels in the uncorrected 6-day velocities have a negative bias. By comparison, the scatterplot of the corrected 6-day velocities, $V_{6 \text { day }}^{c}$, versus the $V_{E P L}$ is centred on the diagonal 1:1 line (Figure 11 (b)) indicating a high-degree of correlation between $V_{6 \text { day }}^{c}$ and $V_{E P L}$ and a dramatic reduction in the phase bias. The coefficient of determination, $\mathrm{R}^{2}$, increases from 0.19 


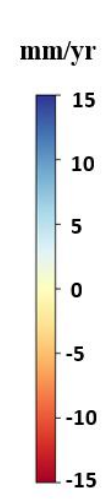

is
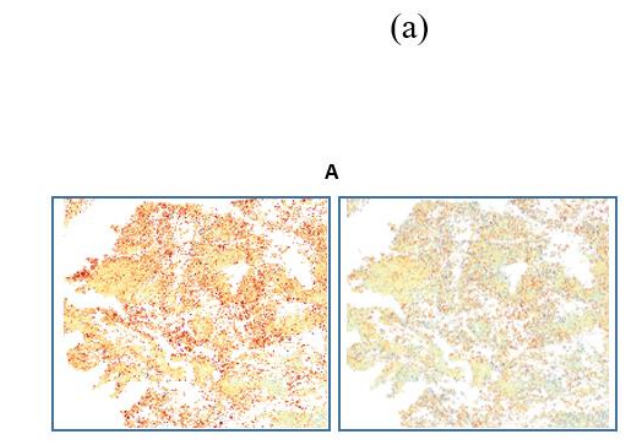

(c)
$V_{E P L}-V_{6 / 12 / 18 d a y}^{c}$

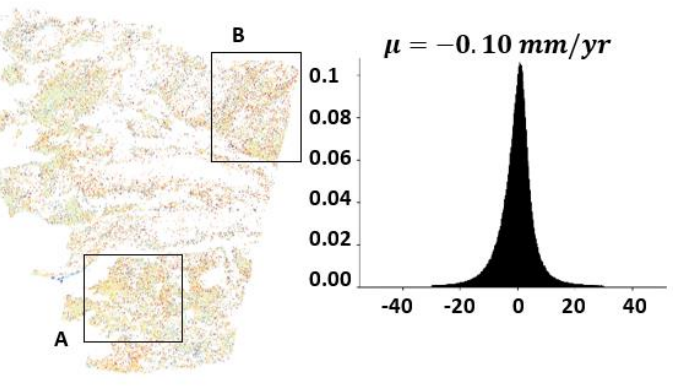

(b)

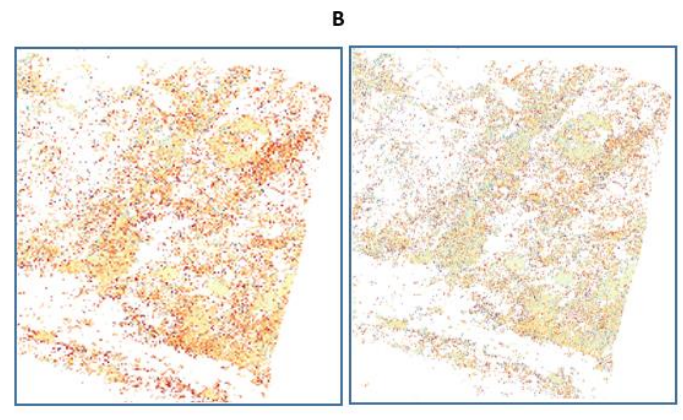

(d)

Figure 13 . The effect of the phase bias correction on the velocity estimation. $V_{E P L}-V_{6 / 12 / 18 \text { day }}$ is shown in (a) and $V_{E P L}-$ $V_{6 / 12 / 18 \text { day }}^{c}$ is shown in (b). The zoomed-in view of the two subsets A and B are shown in panels (c) and (d) respectively.

before the correction to 0.63 after correction, and the RMSE decreased from 11.3 to 6.28 after correcting for the interferograms.

286 Plotting maps of the difference between velocities obtained with EPL and those calculated from 287 our corrected/uncorrected 6-day interferograms (Figure 12) confirms that the corrected velocities 288 are much closer to those from EPL. Comparing the histograms shows that the mean and standard 289 deviation of the differences between the velocities, changes from $-7.08 \pm 8.8 \mathrm{~mm} / \mathrm{yr}$, to $290-0.28 \pm 6.2 \mathrm{~mm} / \mathrm{yr}$ after correction. 
291 In the next experiment, we included all the 6, 12 and 18-day interferograms in our velocity 292 estimation. We calculated velocities using both the original $\left(V_{6 / 12 / 18 \text { day }}\right)$ and the corrected

293 interferograms $\left(V_{6 / 12 / 18 \text { day }}^{c}\right)$ and calculated their difference with $V_{E P L}$ (Figure 13). Using the 294 corrected interferograms decreases the mean velocity bias to $-0.1 \mathrm{~mm} / \mathrm{yr}$.

295 Similar to the velocity estimation using 6-day interferograms only, the scatterplot (Figure 14) 296 shows good correlation between EPL velocities and those estimated from 6/12/18-day 297 interferograms, after correction. The coefficient of determination, $\mathrm{R}^{2}$, increases from 0.63 before 298 the correction to 0.66 after correction, and the RMSE decreased from 6.4 to 6.0 after correcting 299 for the interferograms. Table 1 shows a summary of the average velocities obtained with the 6-day and 6/12/18-day 301 interferograms before and after correction in different land cover classes. The EPL estimated 302 velocities are also given in this table. For all land cover classes, our corrected velocities agree well 303 with those from phase linking approach.

\section{ALL URBAN FOREST CROPLAND}

\begin{tabular}{c|cccc}
\hline $\boldsymbol{V}_{\mathbf{6} \text { day }}$ & 10.8 & 7.1 & 15.0 & 40.2 \\
$\boldsymbol{V}_{\mathbf{6} \text { day }}^{\boldsymbol{c}}$ & 4.0 & 3.5 & 4.5 & 12.8 \\
$\boldsymbol{V}_{\mathbf{6 / 1 2} / \mathbf{1 8} \text { day }}$ & 6.7 & 5.0 & 8.8 & 21.1 \\
$\boldsymbol{V}_{\mathbf{6 / 1 2 / 1 8} \text { day }}$ & 3.8 & 3.5 & 4.3 & 9.2 \\
$\boldsymbol{V}_{\boldsymbol{E} \boldsymbol{P} \boldsymbol{L}}$ & 3.7 & 4.7 & 4.2 & 11.0
\end{tabular}




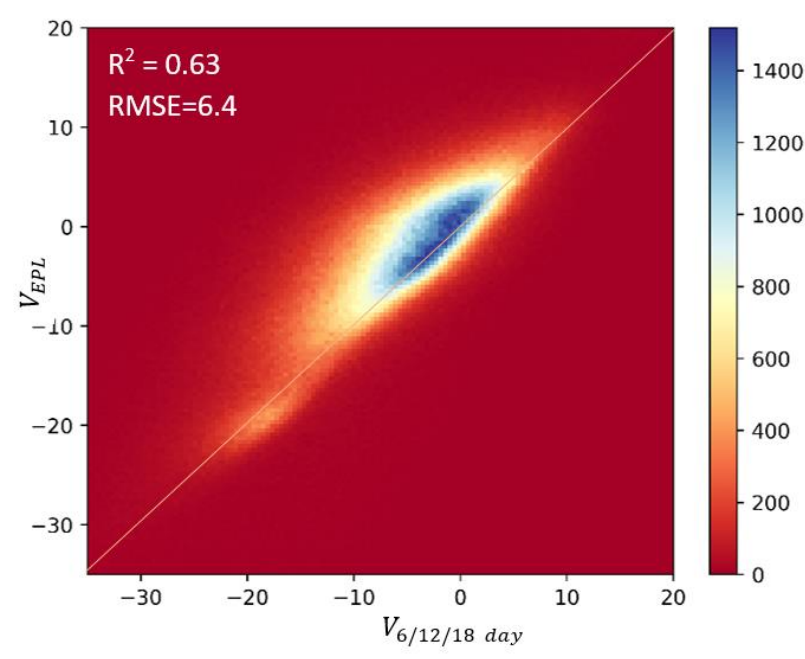

(a)

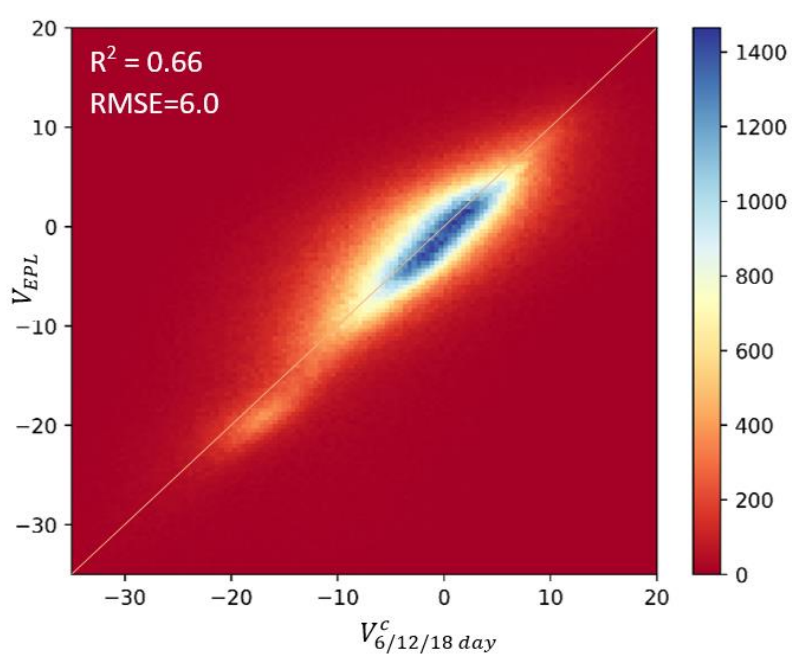

(b)

312 Figure 14) Scatterplot of 360-day velocities obtained from Eigendecomposition Phase Linking $\left(V_{E P L}\right)$ against velocities obtained 314 from (a) uncorrected 6/12/18-day interferograms, $V_{6 / 12 / 18 \text { day }}$, and (b) 6/12/18-day interferograms corrected with our empirical 315 approach $V_{6 / 12 / 18}^{c}$ day

316 Comparing the Figures 12 and 13 reveals that correcting the interferograms using the proposed 317 strategy led to consistent phases. Clearly, upon correcting for the phase bias using the proposed 318 method, it does not make any difference which stack of the interferograms be used for velocity 319 estimation and the 6-day velocity will have very similar performance as the 6, 12 and 18-day 320 velocity. This proves the consistency of the proposed strategy for correcting the phase bias.

\section{Conclusions}

We have shown that short-interval interferograms can be highly affected by phase bias (also known as fading signals) and the accumulation of this phase bias in time can highly affect the estimated

324 velocity. We provide a readily applicable method to estimate the bias corrections for the interferograms. The proposed correction strategy is simple and effective in addressing the phase bias by providing a close performance to the phase linking approach. The method relies on the estimation of two constant regularization parameters, which can easily be calculated using a single long-term interferogram. The proposed method is based on the assumption that the phase bias in 


\section{The paper is a non-peer reviewed EarthArXiv preprint}

an interferogram is linearly related to the sum of the bias shorter interferograms spanning the same time. In this study, we used constant values for $a_{1}$ and $a_{2}$, which relate the biases in 6-day interferograms with those in 12-day and 18-day interferograms. Further investigation is needed to determine if these are universal constants or if they vary spatially. We note that a similar approach could also be developed for areas where the revisit time for Sentinel-1 is 12 days.

Though efficient and robust, PL approaches are computationally expensive both in terms of generating $\mathrm{N}(\mathrm{N}-1) / 2$ interferograms and estimating the $(\mathrm{N}-1)$ optimal phases through often iterative optimizations of the underlying covariance matrix. However, our proposed method only requires calculating $(3 \mathrm{~N}-6)$ interferometric phases and solves for the bias correction using through a single-step, straightforward and inexpensive least square inversion of Equation (10). This is of high importance, particularly for automatic InSAR systems such as COMET-LiCSAR, which are designed to automatically produce InSAR products by processing all Sentinel-1 acquisitions in a frame ( 60 new 6-day acquisitions per frame per year over Europe).

More importantly, the quality of the PL estimated phases highly depends on the availability of the long-term interferograms. In case of the decorrelated regions such as forest or agricultural areas, where long-term coherence is difficult to maintain, the a posteriori coherence is degraded. Our proposed method, on the other hand, is immune to this coherence loss as it only relies on the short term interferograms (6/12/18 day in this study) for estimating the correction terms. We identified a total of 2,400,000 points as coherent pixels (in section 5), whereas this number was decreased to 1,300,000 points when using the EPL approach (in section 6). Therefore, our correction method 


\section{The paper is a non-peer reviewed EarthArXiv preprint}

can be applied to global compilations of short-term interferograms and offers the possibility of accurate long-term velocities without a requirement for coherence in long-term interferograms.

\section{Acknowledgments, Samples, and Data}

This research was supported by the Natural Environmental Research Council (NERC) through COMET. COMET is the UK Natural Environment Research Council's Centre for the Observation and Modelling of Earthquakes, Volcanoes and Tectonics, a partnership between UK Universities and the British Geological Survey. The Sentinel-1 data were obtained via the Copernicus Program of ESA and processed by the COMET-LiCSAR system. LiCSAR uses JASMIN, the UK's collaborative data analysis environment (http://jasmin.ac.uk).

\section{References}

Ansari, H., Zan, F.D., \& Bamler, R. (2018). Efficient Phase Estimation for Interferogram Stacks. IEEE Transactions on Geoscience and Remote Sensing, 56, 4109-4125

Ansari, H., Zan, F.D., \& Parizzi, A. (2021). Study of Systematic Bias in Measuring Surface Deformation With SAR Interferometry. IEEE Transactions on Geoscience and Remote Sensing, 59, 1285-1301

Berardino, P., Fornaro, G., Lanari, R., \& Sansosti, E. (2002). A new algorithm for surface deformation monitoring based on small baseline differential SAR interferograms. IEEE Transactions on Geoscience and Remote Sensing, 40, 2375-2383

Biggs, J., Burgmann, R., Freymueller, J.T., Lu, Z., Parsons, B., Ryder, I., Schmalzle, G., \& Wright, T. (2009). The postseismic response to the 2002 M 7.9 Denali Fault earthquake: constraints from InSAR 2003-2005. Geophysical Journal International, 176, 353-367

Cao, N., Lee, H., \& Jung, H.C. (2016). A Phase-Decomposition-Based PSInSAR Processing Method. IEEE Transactions on Geoscience and Remote Sensing, 54, 1074-1090

De Zan, F., \& Gomba, G. (2018). Vegetation and soil moisture inversion from SAR closure phases: First experiments and results. Remote Sensing of Environment, 217, 562-572

De Zan, F., Parizzi, A., Prats-Iraola, P., \& López-Dekker, P. (2014). A SAR Interferometric Model for Soil Moisture. IEEE Transactions on Geoscience and Remote Sensing, 52, 418-425

De Zan, F., Rocca, F., \& Rucci, A. (2007). PS PROCESSING WITH DECORRELATING TARGETS. In De Zan, F., Zonno, M., \& López-Dekker, P. (2015). Phase Inconsistencies and Multiple Scattering in SAR Interferometry. IEEE Transactions on Geoscience and Remote Sensing, 53, 6608-6616

Farr, T.G., Rosen, P.A., Caro, E., Crippen, R., Duren, R., Hensley, S., Kobrick, M., Paller, M., Rodriguez, E., Roth, L., Seal, D., Shaffer, S., Shimada, J., Umland, J., Werner, M., Oskin, M., Burbank, D., \& Alsdorf, D. (2007). The Shuttle Radar Topography Mission. Reviews of Geophysics, 45

Ferretti, A., Fumagalli, A., Novali, F., Prati, C., Rocca, F., \& Rucci, A. (2011). A New Algorithm for Processing Interferometric Data-Stacks: SqueeSAR. IEEE Transactions on Geoscience and Remote Sensing, 49, 3460-3470 Fornaro, G., Verde, S., Reale, D., \& Pauciullo, A. (2015). CAESAR: An Approach Based on Covariance Matrix Decomposition to Improve Multibaseline-Multitemporal Interferometric SAR Processing. IEEE Transactions on Geoscience and Remote Sensing, 53, 2050-2065

Foroughnia, F., Nemati, S., Maghsoudi, Y., \& Perissin, D. (2019). An iterative PS-InSAR method for the analysis of large spatio-temporal baseline data stacks for land subsidence estimation. International Journal of Applied Earth Observation and Geoinformation, 74, 248-258 


\section{The paper is a non-peer reviewed EarthArXiv preprint}

Goldstein, R.M., \& Werner, C.L. (1998). Radar interferogram filtering for geophysical applications. Geophysical Research Letters, 25, 4035-4038 Guarnieri, A.M., \& Tebaldini, S. (2008). On the Exploitation of Target Statistics for SAR Interferometry Applications. IEEE Transactions on Geoscience and Remote Sensing, 46, 3436-3443

Juncu, D., Árnadóttir, T., Hooper, A., \& Gunnarsson, G. (2017). Anthropogenic and natural ground deformation in the Hengill geothermal area, Iceland. Journal of Geophysical Research: Solid Earth, 122, 692-709 Lazecký, M., Spaans, K., González, P.J., Maghsoudi, Y., Morishita, Y., Albino, F., Elliott, J., Greenall, N., Hatton, E., Hooper, A., Juncu, D., McDougall, A., Walters, R.J., Watson, C.S., Weiss, J.R., \& Wright, T.J. (2020). LiCSAR: An Automatic InSAR Tool for Measuring and Monitoring Tectonic and Volcanic Activity. Remote Sensing, 12 Massonnet, D., Briole, P., \& Arnaud, A. (1995). Deflation of Mount Etna monitored by spaceborne radar interferometry. Nature, 375, 567-570

Michaelides, R.J., Zebker, H.A., \& Zheng, Y. (2019). An Algorithm for Estimating and Correcting Decorrelation Phase From InSAR Data Using Closure Phase Triplets. IEEE Transactions on Geoscience and Remote Sensing, 57, 10390-10397

Morishita, Y., Lazecky, M., Wright, T.J., Weiss, J.R., Elliott, J.R., \& Hooper, A. (2020). LiCSBAS: An OpenSource InSAR Time Series Analysis Package Integrated with the LiCSAR Automated Sentinel-1 InSAR Processor. Remote Sensing, 12

Samiei-Esfahany, S., Martins, J.E., Leijen, F.v., \& Hanssen, R.F. (2016). Phase Estimation for Distributed Scatterers in InSAR Stacks Using Integer Least Squares Estimation. IEEE Transactions on Geoscience and Remote Sensing, 54, 5671-5687

Temtime, T., Biggs, J., Lewi, E., Hamling, I., Wright, T., \& Ayele, A. (2018). Spatial and temporal patterns of deformation at the Tendaho geothermal prospect, Ethiopia. Journal of Volcanology and Geothermal Research, 357 , $56-67$

Walters, R.J., Holley, R.J., Parsons, B., \& Wright, T.J. (2011). Interseismic strain accumulation across the North Anatolian Fault from Envisat InSAR measurements. Geophysical Research Letters, 38

Weiss, J.R., Walters, R.J., Morishita, Y., Wright, T.J., Lazecky, M., Wang, H., Hussain, E., Hooper, A.J., Elliott, J.R., Rollins, C., Yu, C., González, P.J., Spaans, K., Li, Z., \& Parsons, B. (2020). High-Resolution Surface Velocities and Strain for Anatolia From Sentinel-1 InSAR and GNSS Data. Geophysical Research Letters, 47, e2020GL087376

Zwieback, S., Liu, X., Antonova, S., Heim, B., Bartsch, A., Boike, J., \& Hajnsek, I. (2016). A Statistical Test of Phase Closure to Detect Influences on DInSAR Deformation Estimates Besides Displacements and Decorrelation Noise: Two Case Studies in High-Latitude Regions. IEEE Transactions on Geoscience and Remote Sensing, 54, 5588-5601 